\title{
Impact of ED Scribes in a Residency Program
}

Mumtaz Munshi, BS ${ }^{1}$, Daniel Corson-Knowles, MD², Nash Whitaker ${ }^{3}$

${ }^{1}$ Indiana University School of Medicine, ${ }^{2}$ Indiana University School of Medicine, Department of Emergency Medicine

Background and Hypothesis: In the electronic medical record era, clerical documentation has become a significant source of burden in the emergency department (ED). Medical scribes have been advocated as a method to reduce clerical documentation burden while improving physician productivity and satisfaction. The impact of scribes in a non-academic ED setting has been found to be beneficial, and a small survey suggested resident physicians found scribes to be a valuable fatigue-mitigation strategy. The goal of this study was to assess the impact of ED scribes on residents, including time burden of documentation, signals of emotional impact that contribute to burnout, and learning/teaching opportunities.

Experimental Design or Project Methods: This observational time motion analysis assesses scribe impact on emergency medicine residents. Observations were pseudo-randomized in a 1:1 fashion to shifts without or with scribes. Trained observers tracked the activity of upper level residents in the high acuity section of a busy urban county ED for 4 hours of each shift. At the end of each shift, residents self-reported post-shift documentation time and completed a survey assessing residents' perception of their shift and the impact of scribes.

Results: Residents reported that scribes improved their overall experience during their shift. They also reported that scribes reduced documentation time $(0: 11: 33 \pm 0: 04: 24$ v. 0:08:56 $\pm 0: 05: 36)$ and allowed them to better connect with and care more for their patients $(9.6 \pm 0.8$ v. $8.7 \pm 1.3)$.

Conclusion and Potential Impact: Scribes improved emergency medicine residents' perceptions of their educational experience and reduced symptoms of burnout during shifts. 